\title{
Letter to the Editor concerning "Risk factors for sciatica leading to hospitalization" by U. Euro et al. Eur Spine J (2017) doi:10.1007/ s00586-017-5182-8
}

\author{
Tomoyuki Kawada ${ }^{1}$ (D)
}

Received: 23 October 2017 / Accepted: 21 November 2017 / Published online: 27 November 2017

๑) Springer-Verlag GmbH Germany, part of Springer Nature 2017

\section{Dear Editor,}

I read with interest the article by Euro et al. [1], who examined risk factors for sciatica by a prospective study with special reference to hospitalization. Among 13,095 subjects, who were free from low-back disorders at the baseline, 702 incident sciatica cases occurred. Among men, the adjusted hazard ratios (AHRs) [95\% confidence intervals (CIs)] of metal/machine workers and other industrial workers against white-collar workers for sciatica were 2.57 (1.47-4.50) and 1.44 (1.06-1.95), respectively. Among women, AHRs (95\% CIs) of nurses and related occupations, sales workers, and industrial workers for sciatica were 1.81 (1.18-2.78), 1.56 (1.05-2.31), and 1.46 (1.03-2.08), respectively. In contrast, leisure-time physical activity was a protective factor of sciatica among men. I have some concerns about their study.

First, Shiri et al. [2] conducted a meta-analysis to assess the effect of lifestyle factors on hospitalization for sciatica by considering obesity and leisure-time physical activity. AHRs (95\% CIs) of current smoking and walking/cycling to workplace for sciatica were $1.33(1.13-1.56)$ and 0.67 (0.47-0.96), respectively. Euro et al. [1] conducted a stratified analysis by sex, and Shiri et al. [2] used "sex" as an adjusting variable. Euro et al. [1] recognized overweight as a significant risk factor for hospitalization for sciatica among women, and protective effect of leisure-time physical activity on hospitalization for sciatica was observed among men. Sex difference of risk factors should be considered for a meta-analysis.

Second, the authors recognized that health assessment (self-rated health) was significantly associated with

Tomoyuki Kawada

kawada@nms.ac.jp

1 Department of Hygiene and Public Health, Nippon Medical School, 1-1-5 Sendagi, Bunkyo-Ku, Tokyo 113-8602, Japan hospitalization for sciatica among both men and women. As the authors conducted a prospective study, self-rated health could be considered as a good predictor of hospitalization for sciatica. As "average" against "good" self-rated heath was also a risk factor for hospitalization for sciatica, keeping good health is recommended for preventing hospitalization for sciatica.

Finally, there was no precise description on the amount of physical demand in each occupation, and I think that the quantitative evaluation is needed to know dose-response relationship. Although the underlying mechanism on sciatica is complicated [3], occupational workload on sciatica should be quantitatively evaluated by further study.

\section{Compliance with ethical standards}

Conflict of interest No.

Financial disclosure statement There is no financial disclosure in this study.

\section{References}

1. Euro U, Knekt P, Rissanen H, Aromaa A, Karppinen J, Heliövaara M (2017) Risk factors for sciatica leading to hospitalization. Eur Spine J. https://doi.org/10.1007/s00586-017-5182-8

2. Shiri R, Euro U, Heliövaara M et al (2017) Lifestyle risk factors increase the risk of hospitalization for sciatica: findings of four prospective cohort studies. Am J Med. https://doi.org/10.1016/j. amjmed.2017.06.027

3. Kääriä S, Leino-Arjas P, Rahkonen O, Lahti J, Lahelma E, Laaksonen M (2011) Risk factors of sciatic pain: a prospective study among middle-aged employees. Eur J Pain 15:584-590 